\title{
New Technologies in COPD Management
}

\author{
Taylor R. Spencer • Timothy F. Barcomb
}

Published online: 23 July 2014

(c) Springer Science+Business Media New York 2014

\begin{abstract}
Chronic obstructive pulmonary disease (COPD) is a progressive and debilitating disease that affects millions of Americans. Numerous technological advances have been made recently in this field. This article reviews the use of novel technologies in COPD patients, focusing on the medical literature from 2013. These advances affect the diagnosis, the management of acute exacerbations, and the long-term, outpatient care of this condition. Diagnostic advances are examined such as novel applications of computed tomography, plethysmography, and analysis of the breath. Acute interventions include oxygen therapy, extracorporeal gas exchange, and molecular or nanotechnology. Finally, a preponderance of recent literature focuses on telemedicine to facilitate monitoring and treatment. Emerging technologies have a significant potential to positively impact patient care.
\end{abstract}

Keywords COPD $\cdot$ Technology $\cdot$ Pulmonary medicine

\section{Introduction}

Chronic lower respiratory disease, primarily chronic obstructive pulmonary disease (COPD), is the third leading cause of death in USA. Fifteen million Americans report a diagnosis of COPD, with a potentially large number of undiagnosed cases [1]. Technological advances are being used to enhance the diagnosis and management of this condition. This article reviews the 2013 medical literature

T. R. Spencer $(\varangle) \cdot$ T. F. Barcomb

AMC Emergency Medicine Group, 47 New Scotland Avenue, MC 139, Albany, NY 12208, USA

e-mail: spencet@mail.amc.edu that focused on technological advances in diagnosis and treatment of COPD.

A literature search was performed on Medline using the terms "COPD and technology." A total of 117 articles were identified in the date range of January 1, 2013-January 1, 2014. Of the articles identified, 16 were discarded based upon review of the title or abstract alone. The remaining 101 articles were reviewed by one of the two authors to ensure applicability. A total of 33 articles were deemed applicable and incorporated into this review. From these articles' references, an additional 16 articles were identified and incorporated into this review. These constituted either potential relevant articles from 2013, or other heavily cited references preceding 2013. This literature review emphasizes advances in the diagnosis, acute management, and long-term care or outpatient management of COPD.

\section{Diagnosis}

Emerging imaging technology has been applied to COPD diagnosis, with many focusing on the role of computed tomography (CT) scan. Mulshine and colleagues explore high-resolution CT imaging in pulmonary disease, including validating new software against a series of images for which long-term clinical follow-up is known. The authors note that, with this software, high-resolution CT can be applied for an earlier window into COPD progression [2]. An automated emphysema score using principal component analysis with CT shows strong correlations with objective measures of COPD severity and facilitates strong intra- and inter-rater reliability independent of clinical experience in a small study [3]. Thomsen and colleagues also attempt to classify COPD using "causal probabilistic networks," or Bayesian networks. The network they model 
connects radiographic findings, underlying pathophysiology, and clinical effects. Among the pathophysiologic features of this network include ventilation/perfusion changes, in addition to gas exchange and changes in forced expiratory volume (FEV1). The authors reported that "it may be possible to successfully use causal knowledge to relate clinical and physiological data to radiological description when characterizing a patient with COPD." The causal probabilistic network may be an additional diagnostic tool to classify and monitor disease progression without a complete dependence on high-resolution CTessentially predicting imaging results-however, it needs further validation [4].

In the causal probabilistic network designed by Thomsen, the clinical effects of physiologic/radiographic changes in COPD include both symptoms (i.e., productive cough) and spirometric changes (i.e., FEV1) [4]. Mirsadraee and colleagues, however, proposed that spirometry alone may be used in a unique way for earlier COPD identification. The authors note that ratio between maximum mid-expiratory flow and forced vital capacity (MMEF/FVC) in the group at risk of COPD is significantly lower than in normal controls. The ratio is further reduced in patients with a COPD diagnosis, correlating with COPD stages. Early COPD (smoker subjects without other spirometric derangement) might be identified by MMEF/FVC [5]. Thus, spirometry is a technology that could enhance early diagnosis. Spirometry may also be a potent prognosticator. Leivseth and colleagues reviewed the spirometry classifications endorsed by the Global Initiative for Chronic Obstructive Lung Disease (GOLD). This is compared with the $\mathrm{ABCD}$ grouping, which combines symptom burden and risk of exacerbations into one of 4 groups (labeled "A", "B", "C", or "D"). In the Nord-Trøndelag Health Study (HUNT) Lung Study-a large longitudinal population health study in Norway - the spirometric GOLD grades predict mortality better than clinical $\mathrm{ABCD}$ grouping [6 $\left.6^{\bullet}\right]$. Many technologic advances may enhance prognostication in COPD, but must be balanced with cost considerations. Such considerations have not been explored in the reviewed literature. However, spirometry is inexpensive, patientfriendly, and easily interpreted. This holds promise not just due to its potent prognostication potential, but also for its efficient use of resources.

Outside of the more traditional modalities of CT and spirometry, other emerging technologies have been explored. Using bronchoscopy, fiber-optic color Doppler optical coherence tomography (CDOCT) has been used to map the in vivo 3-dimensional vascular network of the lung. Ventilation-perfusion mismatch is a hallmark of COPD. The perfusion changes in COPD, angiogenesis, and vascular remodeling may be seen with this developing technology [7].
Plethysmography measures changes in volume within an organ and, therefore, is useful for identifying the structural changes of the lung associated with COPD. The whole-body plethysmograph consists of a rigid chamber in which the subject sits, while breathing through an apparatus recording the rate of airflow to and from the lungs. Pressure differences are measured with transducers [8]. Plethysmography identifies lung hyperinflation and measures bronchodilator effects. It has also been used in research to show a treatment effect using $\mathrm{N}$-acetyl cysteine to enhance antimuscarinic bronchodilators [9]. Additionally, plethysmography has been used in studies showing an association between adiponectin and peripheral airway obstruction or response to steroids [10]. Others have used it to explore the physiologic responses to phosphodiesterase 4 [11], or nasal positive expiratory pressure in patients with COPD [12]. Many of these recent studies supplement results with data from forced oscillation techniques (FOT), in particular impulse oscillometry. This is a passive measurement complimentary to conventional pulmonary function testing. Pressure oscillations are applied at the mouth to measure pulmonary resistance and reactance. In adults, relative to spirometry or plethysmography, FOT's diagnostic value in COPD remains controversial according to Schulz and colleagues. Their recent research attempts to clarify age-related reference ranges to enable potential future applications of this diagnostic modality in clinical practice [13]. These technologies still lack standardized clinical applications.

There is an emerging body of the literature that explores the diagnostic potential of a patient's breath as a noninvasive, radiation-free test. A recent review summarizes the electronic nose (e-nose) technology in respiratory medicine. The e-nose is an artificial sensor designed to identify selective patterns of exhaled volatile organic compounds (VOCs). These serve as a marker of underlying lung inflammation or pathophysiology. Variations of the e-nose may use acoustic, optical, or calorimetric sensors. Other iterations of the e-nose also include mass spectrometry, ion mobility spectrometry gas chromatography, infrared spectrometry, or selected ion flow tube mass spectrometry (SIFT-MS). However, this technology is evolving and requires improved standardization, enhanced specificity and sensitivity, and further validation for clinical practice. Sensors are generally designed to identify patterns of VOCs ("breathprints") rather than measuring specific organic compounds, whereas others are working to identify the complete patterns of biomolecules ("breathome") [14••].

E-nose breathprints correlate strongly with airway inflammation, as identified by measuring sputum inflammatory markers [15]. Montuschi and colleagues' cited recent evidence of an e-nose identifying unique patterns to distinguish COPD from asthma [14*0]. The e-nose was able to discriminate mild to severe asthma from COPD with 
$96 \%$ accuracy. But it was only $66 \%$ accurate in distinguishing COPD from healthy smokers [16]. It is hypothesized that this may represent a partial overlap because of a subset of smokers who are symptom free, but at increased risk of COPD. There is even evidence that the e-nose can distinguish between stages of COPD, based upon GOLD staging [15].

Others have tried to take this a step further studying a lower-cost prototype, a simple e-nose using 6 semiconductor sensors. Before testing on patients, they have tested the ability of the simple e-nose to distinguish between reference mixtures containing COPD markers $\mathrm{N}, \mathrm{N}$ dimethylformamide and $N, N$-dimethylacetamide. Mixtures with a total of 5-15 different VOC were tested (notably, human breath contains more than 200 VOC compounds). The simple e-nose worked with limited success in the experimental mixtures. The concentration of components-not the number of VOCs-is most important in influencing the e-nose's ability to differentiate mixtures. Based on current studies, this simple e-nose prototype would not yet have capacity for early COPD identification [17].

While the e-nose assesses VOCs, exhaled breath may provide other diagnostic information. Kubán and Foret recently explored the applications of exhaled breath condensate (EBC). Rather than focusing on the VOCs, this research focuses on the aqueous part of the breath, which contains mainly nonvolatile compounds and water-soluble volatiles. Pulmonary inflammation in COPD produces mediators of oxidative stress that are released into the airway lining fluid (ALF). Specimens are traditionally obtained by bronchoalveolar lavage or sputum induction. Alternatively, the ALF from central airways is aerosolized into the EBC. This technique has the advantage of being a noninvasive means of specimen collection. Multiple different sampling devices and techniques are in practice without standardization. In their review, Kubán and Foret postulate potential roles in COPD management including identifying individuals with increased susceptibility for COPD, estimating degree of lung inflammation, and evaluating response to therapy. They cite increased levels of nitrite, prostaglandin E2, or $S$-nitrosothiols in COPD. Some biomarkers in the EBC may distinguish COPD from asthma or other pulmonary pathology [18••]. Using nuclear magnetic resonance-based metabolomics analysis of EBC can also distinguish COPD from other conditions [19]. And Fens and colleagues used it as one part of an analysis, finding that "functional, clinical, radiological, and exhaled breath metabolomic features identify subphenotypes of COPD in a community-based population of heavy (ex-) smokers," with an accuracy of $97.4 \%$ [20•]. Leukotriene B4 is the only biomarker identified in EBC that increases in the visit prior to a COPD exacerbation, potentially predicting these exacerbations [21]. In exacerbations themselves, a significant increase in cytokines IL1 $\beta$, IL-6, IL-8, and IL-10 occurs relative to chronic COPD. EBC can further demonstrate physiologic responses to therapy. Some cytokines such as IL- 8 are reduced in EBC when COPD is treated with steroids $[18 \bullet \bullet]$. Thus, much like the e-nose to assess VOCs, the EBC is an emerging technology that may eventually facilitate diagnosis, assess inflammatory response/oxidative stress, identify COPD exacerbations, and evaluate response to therapy. This technology requires further validation and exploration to determine its optimal use before it can become more broadly used in clinical medicine.

Lastly, a subset of articles identified in the literature review fell in the domain of molecular biology. The vast majority of these are not clinically relevant, with no significant diagnostic, prognostic, or therapeutic utility. Given the nature of this research, it may take years for molecular breakthroughs to find their way into clinical practice. But research with promise includes a study by Kelly and colleagues. The authors used inflammatory biomarkers to predict mortality in COPD. Several serum inflammatory biomarkers were studied. C-reactive protein, IL-6, pulmonary and activation-regulated chemokine, and fibronectin: C-reactive protein ratio were each independently associated with increased risk of death. Only IL-6 was shown to further predict mortality when combined with known clinical variables such as the BMI, obstruction, dyspnea, and exercise capacity index [22]. Even in the realm of molecular biology, these discoveries may someday provide prognostic value for patients in COPD.

Clearly, the available literature from 2013 reflects the breadth of technologies that may impact the diagnosis of COPD. The technologies being used range from expansion of common tools such as CT or spirometry, to novel and emerging options such as plethysmography, breath analysis, or molecular biology. Given the financial limitations of the healthcare system, and the fact that many of these diagnostic modalities are expensive and still largely unproven, restraint is warranted. While these technologies are interesting, they must be cost-effective and demonstrate patient-oriented beneficial outcomes to merit widespread implementation.

\section{Acute Management}

A second application for technology in COPD has been for the acute medical management. Technological advances may be useful for impaired oxygenation. For example, a novel prototype pulse oximeter may have utility in outpatient monitoring. The device uses vertical cavity surface emitting laser (VCSEL) technology, which is lower power, 
low cost, and able to be worn behind the ear (like a hearing aid) for long-term continuous use [23]. While this device may monitor oxygenation, others have attempted to clarify the best methods for maintaining oxygen saturation. An open cross-sectional crossover study compares continuous oxygen flow by standard nasal cannula (CFNC) with either a pendant reservoir cannula (PRC) that stores oxygen during exhalation, or pulse demand oxygen delivery (DOD) system with a preset bolus of oxygen during early inspiration. In COPD patients, desaturation during a 6-minute walk test is corrected in $79 \%$ of patients by the CFNC or DOD, and in $86 \%$ of patients with PRC. The percentage of patients demonstrating a correction is not a statistical significant difference between these devices, however. The authors further note that, since correction is not achieved in about $20 \%$ of patients, individualized adjustments in severe COPD are crucial [24]. Factors such as the type of portable oxygen concentrator used [25] or the method of oxygen transport might enhance or mitigate the effects of this intervention [26]. Using these currently available technologies appropriately is important for optimal COPD management.

Airway management in COPD has also been a target for technology. A recent retrospective observational study explored the evolving use of noninvasive mechanical ventilation (NIV) for COPD. NIV use increased over time to $12.1 \%$ of COPD patients; however, the rate of intubation did not significantly change over the 12-year study period. Perhaps NIV did not replace intubation, but seems to be used in marginal patients who would not have reached the threshold for intubation before the introduction of NIV. In this population, NIV did not affect the overall mortality [27]. "Despite the continued improvements in the administration of noninvasive ventilation (NIV), refractory hypercapnia and hypercapnic acidosis continue to prevent its successful use in many patients" [28••]. Lund and Federspiel reviewed the potential role of partial extracorporeal $\mathrm{CO} 2$ removal $(\mathrm{ECCO} 2 \mathrm{R})$ in these situations. Unlike extracorporeal membrane oxygenation, or ECMO, $\mathrm{ECCO} 2 \mathrm{R}$ is an adjunct to mechanical ventilation. ECCO2R is used primarily to enhance $\mathrm{CO} 2$ removal rather than for oxygenation. [28*0]. A study of ECCO2R in 21 patientsincluding 14 with COPD exacerbation-notes a high rate of avoiding mechanical ventilation, plus significant corrections of $\mathrm{pH}, \mathrm{pCO} 2$, and respiratory rate. There is no mortality effect, however [29]. A second smaller six patient study of ECCO2R also demonstrates that respiratory rate decreases in conjunction with enhanced $\mathrm{CO} 2$ removal in patients who failed NIV [30]. A pilot study has further suggested that support with partial ECCO2R after intubation may provide more clinical benefit if applied early, before a COPD patient becomes ventilator dependent [31]. And a case report describes avoidance of intubation during acute COPD exacerbation for a lung transplant candidate using ECCO2R [32]. Given the cited risks of mechanical ventilation, including barotrauma/volutrauma or pneumonia, studies suggest ECCO2R may provide an alternative-and perhaps safer-technology for ventilation support [28••].

For patients who do require endotracheal intubation, the Auto-Trak $^{(\mathrm{TM})}$ system is technology which automatically adjusts the triggering and cycling mechanisms during pressure support ventilation. In a recent study of twelve non-COPD volunteers on pressure support ventilation via an oral endotracheal tube, this technology is compared to conventional settings. However, there are no differences in ventilator asynchrony or discomfort. In a complementary bench study modeling COPD, on the other hand, there is a greater triggering delay with the Auto-Trak system [33]. Triggering delay represents the gap between initiation of patient respiratory effort to onset of the ventilator-delivered breath, and such a delay is generally considered undesirable. The Auto-Trak ${ }^{(\mathrm{TM})}$ system may not be beneficial in COPD patients.

A different target of recent investigation is the molecular biology of Chlamydophila pneumonia, a gram-negative bacteria known to cause community-acquired pneumonia and chronic infections in COPD. Ravindranath and colleagues used an in silico synteny-based comparative genomics analysis of $C$. pneumonia and eight other Chlamydial organisms. They determined which Chlamydial organisms have an association with COPD pathogenesis and exacerbation in humans. These data have the potential to identify protein targets for future drug design [34]. Thus, developments in the realm of molecular biology and related technology may someday impact COPD management.

Finally, nanotechnology has promise for the future delivery of therapies for COPD. In a review by Thorley and Tetley on the use of nanomedicines for treatment of pulmonary diseases, it is noted that the lung is a particularly appealing target for nanomedicine. It has large surface area, affords the ability to self-administer in a noninvasive fashion, and avoids first pass metabolism. This allows for local pulmonary benefit and provides an excellent vector for systemic absorption. While many drugs currently are micron-sized, in theory a reformulation to nano-sized could result in deeper penetration and reduced clearance by macrophages. This may potentially lead to increased absorption of pulmonary drugs, with more consistent effects for COPD patients in particular [35॰].

In summary, the recent literature provides much promise for acute COPD management. Technological advances in oxygen delivery, $\mathrm{CO} 2$ removal, and airway management have been recent foci of the literature. But the future of COPD management may lay in molecular biology and nanotechnology. Nanomedicine has many theoretical 
advantages and much promise, but human trials to definitively demonstrate benefit are lacking.

\section{Long-Term/Outpatient Management}

The recent literature on technology in COPD is not limited to the acute phase of the disease. Outpatient and long-term management are also benefiting from developments. Parker and colleagues have planned a randomized, cluster design trial on the translation of COPD guidelines into primary care practice, which is currently underway. Three components of the study were developed based upon an initial needs assessment: a computerized patient activation tool for spirometry, a Web-based COPD guidelines tool for provider decision support, and a COPD patient education toolkit. They plan to evaluate the effectiveness of this multimodal intervention using technology-supported health care. At this time, the study results are pending [36].

Perhaps the most prominent focus for technology in the recent literature has been the emerging applications of telemedicine. The American Telemedicine Association defines telemedicine as "the use of medical information exchanged from one site to another via electronic communications to improve a patient's clinical health status" [37]. This can occur through the e-mail, text messaging, and video conferencing. One common approach is a symptom diary, allowing healthcare providers to track the frequency of COPD exacerbations and identify temporal or behavioral correlations. To facilitate this, patients learn how to perform self-assessments. Data may include vital signs, step counters, home exercise, or portable at-home spirometry. Results can be transmitted to their physician via smartphone [38]. The chronic nature of COPD affords long-standing opportunities to apply telemedicine in outpatient management. The use of telemedicine may spare the cost and time burdens of frequent physician office visits. Outside of acute exacerbations, many adjustments in therapy can be made based on the patient's history and recorded telemedicine data. Telemedicine may foster increased patient confidence in their self-management and bring "peace of mind" to COPD patients, who view this technology to be a legitimate contact with healthcare professionals [39].

The use of telemedicine to facilitate care is frequently studied in conjunction with other chronic diseases, including diabetes, congestive heart failure, hypertension, and asthma [40, 41•, 42-44]. Like COPD, the chronicity of these conditions allows for home monitoring and self-management. Several longitudinal studies combined educational programs with telemedicine and most, though not all, were deemed successful by the authors. As an adjunct for the treatment of COPD specifically, hospital admissions or office visit rates were reduced in COPD patients participating in telemedicine programs in multiple recent studies [41•, 45, 46•]. Baker and colleagues performed a retrospective matched cohort study and found that telemedicine is associated with fewer COPDrelated admissions for Medicare patients [41•]. Telemedicine may enable early recognition of exacerbations, timelier interventions, decreased peak symptom severity, and fewer hospitalizations.

Historically, the goals of COPD treatment have been to better monitor and control symptoms while simultaneously decreasing unanticipated medical encounters (hospitalizations, emergency department visits, and unplanned office visits). These goals should be based on patient-centered characteristic that support "pulmonary rehabilitation at homes of older adults with COPD based on their values, needs, and preferences" [47]. To achieve this, telemedicine communicates information not only from the patient to their primary care provider (PCP), but also from the PCP to a specialist as needed. Thijssing and colleagues made use of telepulmonology consultations to obtain pulmonologist interpretation of spirometry. This successfully avoided specialist referral $21 \%$ of the time. However, the telepulmonology consultations led to a physical referral for $12 \%$ of the consultation where the original PCP intent had been simply to seek advice. Thus, using this identifies patients likely to benefit from formal pulmonology evaluation who otherwise would not have received a referral in the absence of telemedicine [46•].

Patients discharged from the hospital with COPD are susceptible to readmission, and reimbursement for these readmissions has been scrutinized by third-party payers. In recent Danish trial, COPD patients with exacerbation were randomized to receive standard in-hospital treatment or the same treatment at home via telemedicine. The technology included video conferencing and monitoring equipmentspirometer, pulse oximeter, and thermometer [48]. Results have not yet been published; however, if comparable efficacy is seen, this may prompt a paradigm shift not only with how COPD exacerbations are treated, but where that treatment will occur. Applying technology to ensure the same level of care while avoiding readmission would be financially desirable to hospitals, and likely favorable to patients as well.

Patient satisfaction is an important part of the medical encounter, but particularly with telemedicine. As an alternative or adjunct to hospitalization or the office visit, patient preference and satisfaction is presumably essential for the vast majority of telemedicine participation. Overall, patients are satisfied with the use of telemedicine and many feel it empowers them to perform home monitoring [42, 44, 49-52]. But this is not universal. Patients performing telerehabilitation exhibit four types of attitudes depending on their physical and emotional state: indifference, learning as 
part of situations in everyday life, feeling of security, and motivation for performing physical training [52]. This patient motivation may improve health-related quality of life (HRQol), and thus satisfaction. Several nonpharmacological therapies and technologies have proven effective in COPD [49]. Malnourished COPD patients demonstrate decreased exercise capacity, accelerated by smoking. Internet programs, periodic e-mails, and text messages can improve HRQoL by reminders about appointments, assistance with medication compliance, advice on healthy dietary choices, or smoking cessation $[49,53]$.

Physicians are also now recognizing that different patients prefer different technology. A study in the Netherlands found that COPD patients prefer notebook computers to smartphones for telemedicine interactions due to the larger screen size [50]. And, using other measures of patient-reported outcomes, evidence shows that the reports from these technologic alternatives might be interchangeable. For example, the Patient-Reported Outcomes Measurement Information System (PROMIS) scales can be used for COPD, in addition to conditions ranging from rheumatoid arthritis to depression. This survey explores physical function, fatigue, and depression. The tool may be administered by paper or using an alternative technology, including interactive voice response, personal digital assistant, or personal computer. For COPD, research shows no statistically significant difference based upon method of administration. Thus, these alternative technologies may be reasonable methods to measure patient-reported outcomes for clinical research and clinical practice [54]. It is possible that adapting to patient preferences might enhance patient compliance with interventions.

While telemedicine does hold promise for the future of COPD outpatient management, though, there remains a paucity of randomized controlled trials in the recent literature. Few papers reviewed were designed to prove its efficacy versus standard therapy. Despite the inklings of promise suggested by the recent articles, some have questioned the benefit. Bardsley and colleagues did not find a difference in the level of contact with primary care in a randomized trial comparing telemedicine to usual care in English patients with COPD, heart failure, or diabetes [40]. Nguyen and colleagues assessed the perception of dyspnea with activity and found that an Internet-based program did not reduce this symptom compared with usual care [55]. Studies such as these and the limited nature of the available literature have led the Global initiative for chronic obstructive lung disease (GOLD) to have reservations and determine that "accumulating data from a variety of studies indicate that telehealth in any of its current forms has not shown benefits for patients with COPD; thus, telehealth is not recommended for use with COPD patients" [56••]. Clearly, controversy remains regarding the health benefits of this emerging technology in long-term and outpatient care. Perhaps the benefit is not in mortality or objective health measures, so much as providing a viable, noninferior alternative to inpatient management.

\section{Conclusion}

COPD is a debilitating progressive, condition with a significant burden for patients and the healthcare system. Technological advances are being pioneered to enhance the diagnosis and management of this condition. The breadth of the advances seen in the medical literature from the past year is dramatic - from a range of imaging technologies to facilitate diagnosis or prognosis, to the molecular level with the potential to tailor drugs to proteins, to oxygen monitoring and delivery devices, to large-scale telecommunication systems integrating into outpatient monitoring and care. In the domains of diagnosis, acute management, and outpatient or long-term care, the successful integration of effective technologies has the potential to revolutionize and optimize the medical care for patients with COPD. However, many questions still remain regarding which technologies are effective, and how best to integrate these into patient care. These and related questions merit further exploration and will be a promising field of research for years to come.

\section{Compliance with Ethics Guidelines}

Conflict of Interest Taylor R. Spencer and Timothy F. Barcomb have reported no conflict of interest.

Human and Animal Rights and Informed Consent This article does not contain any studies with human or animal subjects performed by any of the authors.

\section{References}

Papers of particular interest, published recently, have been highlighted as:

- Of importance

•. Of major importance

1. "What is COPD?" Centers for disease control and prevention. 13 November 2013. Accessed online at http://www.cdc.gov/copd/ on 7 April 2014.

2. Mulshine JL, Avila R, Yankelevitz D, Baer TM, Estepar RS, Fenton L, Aldige CR. Application of high-resolution CT imaging data to lung cancer drug development: measuring progress: workshop IX. J Thorac Oncol. 2013;8(11):1352-5.

3. Owrangi AM, Entwistle B, Lu A, Chiu J, Hussain N, EtemadRezai R, Parraga G. Semi-automated scoring of pulmonary emphysema from X-ray CT: trainee reproducibility and accuracy. Eur J Radiol. 2013;82(11):e734-41. 
4. Thomsen LP, Weinreich UM, Karbing DS, Helbo Jensen VG, Vuust M, Frøkjær JB, Rees SE. Can computed tomography classifications of chronic obstructive pulmonary disease be identified using Bayesian networks and clinical data? Comput Methods Progr Biomed. 2013;110(3):361-8.

5. Mirsadraee M, Boskabady MH, Attaran D. Diagnosis of chronic obstructive pulmonary disease earlier than current global initiative for obstructive lung disease guidelines using a feasible spirometry parameter (maximal-mid expiratory flow/forced vital capacity). Chron Respir Dis. 2013;10(4):191-6.

6. - Leivseth L, Brumpton BM, Nilsen TI, Mai XM, Johnsen R, Langhammer A. GOLD classifications and mortality in chronic obstructive pulmonary disease: the HUNT Study, Norway. Thorax. 2013;68(10):914-21. In this large study, spirometric GOLD grades predicted mortality better than the $A B C D$ groups in $C O P D$, arguing the prognostic value of this simple diagnostic testing.

7. Lee AM, Ohtani K, Macaulay C, McWilliams A, Shaipanich T, Yang VX, Lam S, Lane P. In vivo lung microvasculature visualized in three dimensions using fiber-optic color Doppler optical coherence tomography. J Biomed Opt. 2013;18(5):50501.

8. Konstantinos Katsoulis K, Kostikas K, Kontakiotis T. Techniques for assessing small airways function: possible applications in asthma and COPD. Respir Med. 2013. doi10.1016/j.rmed.2013. 05.003 .

9. Sinojia R, Shaikh M, Kodgule R, Bhosale S, Madas S, Vaidya A, Salvi S, Brashier B. Priming of beta-2 agonist and antimuscarinic induced physiological responses induced by $1,200 \mathrm{mg} /$ day NAC in moderate to severe COPD patients: a pilot study. Respir Physiol Neurobiol. 2014;15(191):52-9.

10. Leivo-Korpela $\mathrm{S}$, Lehtimäki L, Vuolteenaho $\mathrm{K}$, Nieminen R, Kööbi L, Järvenpää R, Kankaanranta H, Saarelainen S, Moilanen E. Adiponectin is associated with dynamic hyperinflation and a favourable response to inhaled glucocorticoids in patients with COPD. Respir Med. 2014;108(1):122-8.

11. Watz H, Mistry SJ, Lazaar AL, IPC101939 investigators. Safety and tolerability of the inhaled phosphodiesterase 4 inhibitor GSK256066 in moderate COPD. Pulm Pharmacol Ther. 2013; 26(5):588-95.

12. Wibmer T, Rüdiger S, Heitner C, Kropf-Sanchen C, Blanta I, Stoiber KM, Rottbauer W, Schumann C. Effects of nasal positive expiratory pressure on dynamic hyperinflation and six minute walk test in patients with COPD. Respir Care. 2014. doi:10.4187/ respcare.02668.

13. Schulz H, Flexeder C, Behr J, Heier M, Holle R, Huber RM, Jörres RA, Nowak D, Peters A, Wichmann HE, Heinrich J, Karrasch S, KORA Study Group. Reference values of impulse oscillometric lung function indices in adults of advanced age. PLoS One. 2013;8(5):e63366.

14. • Montuschi P, Mores N, Trové A, Mondino C, Barnes PJ. The electronic nose in respiratory medicine. Respiration. 2013;85(1): 72-84. This is a recent review of how the identification of selective patterns of volatile organic compounds in exhaled breath could be used as a biomarker of inflammatory lung diseases.

15. Fens N, de Nijs SB, Peters S, Dekker T, Knobel HH, Vink TJ, Willard NP, Zwinderman AH, Krouwels FH, Janssen HG, Lutter $\mathrm{R}$, Sterk PJ. Exhaled air molecular profiling in relation to inflammatory subtype and activity in COPD. Eur Respir J. 2011;38(6):1301-9.

16. Fens N, Zwinderman AH, van der Schee MP, de Nijs SB, Dijkers E, Roldaan AC, Cheung D, Bel EH, Sterk PJ. Exhaled breath profiling enables discrimination of chronic obstructive pulmonary disease and asthma. Am J Respir Crit Care Med. 2009;180(11): 1076-82.

17. Dymerski T, Gębicki J, Wiśniewska P, Sliwińska M, Wardencki W, Namieśnik J. Application of the electronic nose technique to differentiation between model mixtures with COPD markers. Sensors (Basel). 2013;13(4):5008-27.

18. •• Kubáň P, Foret F. Exhaled breath condensate: determination of non-volatile compounds and their potential for clinical diagnosis and monitoring. A review. Anal Chim Acta. 2013;805:1-18. This review article focuses mainly on the progress in determination of non-volatile compounds in exhaled breath condensate, with an exploration of the clinical relevance.

19. de Laurentiis G, Paris D, Melck D, Montuschi P, Maniscalco M, Bianco A, Sofia M, Motta A. Separating smoking-related diseases using NMR-based metabolomics of exhaled breath condensate. J Proteome Res. 2013;12(3):1502-11.

20. - Fens N, van Rossum AG, Zanen P, van Ginneken B, van Klaveren RJ, Zwinderman AH, Sterk PJ. Subphenotypes of mildto-moderate COPD by factor and cluster analysis of pulmonary function, CT imaging and breathomics in a population-based survey. COPD. 2013;10(3):277-85. This study shows that COPD phenotype can be clustered using functional, clinical, radiological and exhaled breath features with a high degree of accuracy.

21. Tufvesson E, Ekberg M, Bjermer L. Inflammatory biomarkers in sputum predict COPD exacerbations. Lung. 2013;191(4):413-6.

22. Kelly E, Owen CA, Pinto-Plata V, Celli BR. The role of systemic inflammatory biomarkers to predict mortality in chronic obstructive pulmonary disease. Expert Rev Respir Med. 2013; 7(1):57-64

23. Kollmann D, Hogan WK, Steidl C, Hibbs-Brenner MK, Hedin DS, Lichter PA. VCSEL based, wearable, continuously monitoring pulse oximeter. Conf Proc IEEE Eng Med Biol Soc. 2013;2013:4156-9.

24. Martí S, Pajares V, Morante F, Ramón MA, Lara J, Ferrer J, Güell MR. Are oxygen-conserving devices effective for correcting exercise hypoxemia? Respir Care. 2013;58(10):1606-13.

25. Latta TN, Pohle-Krauza RJ, McCarroll ML, Pannikottu K, DiNuoscio DR, Volsko TA. Gothard MD, Krauza ML. Six-minute walk distance is reduced by use of roller and shoulder sling, but not backpack style portable oxygen carriers in patients with COPD. 2012. Submitted for publication to Proceedings of the American Thoracic Society. Accessed online at http://www. atsjournals.org/doi/abs/10.1164/ajrccm-conference.2013.187.1_ MeetingAbstracts.A5121.

26. Leblanc CJ, Lavallée LG, King JA, Taylor-Sussex RE, Woolnough A, McKim DA. A comparative study of 3 portable oxygen concentrators during a 6-minute walk test in patients with chronic lung disease. Respir Care. 2013;58(10):1598-605.

27. Carpe-Carpe B, Hernando-Arizaleta L, Ibáñez-Pérez MC, Palomar-Rodríguez JA, Esquinas-Rodríguez AM. Evolution of the use of noninvasive mechanical ventilation in chronic obstructive pulmonary disease in a Spanish region, 1997-2010. Arch Bronconeumol. 2013;49(8):330-6.

28. • Lund LW, Federspiel WJ. Removing extra CO(2) in COPD patients. Curr Respir Care Rep. 2013;2(3):131-8. This review focuses on recent literature on the use of a novel therapy for removing excess $\mathrm{CO} 2$ in patients experiencing an acute exacerbation of COPD.

29. Kluge S, Braune SA, Engel M, Nierhaus A, Frings D, Ebelt H, Uhrig A, Metschke M, Wegscheider K, Suttorp N, Rousseau S. Avoiding invasive mechanical ventilation by extracorporeal carbon dioxide removal in patients failing noninvasive ventilation. Intensive Care Med. 2012;38(10):1632-9.

30. Spinelli E, Crotti S, Zacchetti L, Bottino N, Berto V, Russo R, Chierichetti M, Protti A, Gattinoni L. Effect of extracorporeal $\mathrm{CO} 2$ removal on respiratory rate in spontaneously breathing patients with chronic obstructive pulmonary disease exacerbation. Crit Care. 2013;17(Suppl 2):P128.

31. Burki NK, Mani RK, Herth FJ, Schmidt W, Teschler H, Bonin F, Becker H, Randerath WJ, Stieglitz S, Hagmeyer L, Priegnitz C, 
Pfeifer M, Blaas SH, Putensen C, Theuerkauf N, Quintel M, Moerer O. A novel extracorporeal $\mathrm{CO}(2)$ removal system: results of a pilot study of hypercapnic respiratory failure in patients with COPD. Chest. 2013;143(3):678-86.

32. Bonin F, Sommerwerck U, Lund LW, Teschler H. Avoidance of intubation during acute exacerbation of chronic obstructive pulmonary disease for a lung transplant candidate using extracorporeal carbon dioxide removal with the Hemolung. J Thorac Cardiovasc Surg. 2013;145(5):e43-4.

33. Vasconcelos RD, Melo LH, Sales RP, Marinho LS, Deulefeu FC, Reis RC, Alves-de-Almeida M, Holanda MA. Effect of an automatic triggering and cycling system on comfort and patientventilator synchrony during pressure support ventilation. Respiration. 2013;86(6):497-503.

34. Ravindranath BS, Krishnamurthy V, Krishna V, SK C. In silico synteny based comparative genomics approach for identification and characterization of novel therapeutic targets in Chlamydophila pneumoniae. Bioinformation. 2013;9(10):506-10.

35. - Thorley AJ, Tetley TD. New perspectives in nanomedicine. Pharmacol Ther. 2013;140(2):176-85. This review of the recent advances in nanotechnology for therapeutic applications has an emphasis on the potential applications with the respiratory system, although much remains to be determined before any widespread application would be feasible.

36. Parker DR, Eaton CB, Ahern DK, Roberts MB, Rafferty C, Goldman RE, McCool FD, Wroblewski J. The study design and rationale of the randomized controlled trial: translating COPD guidelines into primary care practice. BMC Fam Pract. 2013; 6(14):56.

37. "What is Telemedicine?" American Telemedicine Association. 2102. Accessed online at http://www.americantelemed.org/abouttelemedicine/what-is-telemedicine\#.UOVFdsaBX1o on 7 April 2014.

38. Işik AH, Güler I, Sener MU. A low-cost mobile adaptive tracking system for chronic pulmonary patients in home environment. Telemed J E Health. 2013;19(1):24-30.

39. Gale N, Sultan H. Telehealth as 'peace of mind': embodiment, emotions and the home as the primary health space for people with chronic obstructive pulmonary disorder. Health Place. 2013;21:140-7.

40. Bardsley M, Steventon A, Doll H. Impact of telehealth on general practice contacts: findings from the whole systems demonstrator cluster randomised trial. BMC Health Serv Res. 2013;8(13): 395.

41. - Baker LC, Macaulay DS, Sorg RA, Diener MD, Johnson SJ, Birnbaum HG. Effects of care management and telehealth: a longitudinal analysis using Medicare data. J Am Geriatr Soc. 2013;61(9):1560-7. This study raises the potential cost and health outcomes benefits of telemedicine for chronic conditions in a relatively large retrospective matched cohort study. These arguments are primary drivers of the push for telemedicine.

42. Lamothe L, Paquette MA, Fortin JP, Labbé F, Messikhs D, Duplantie J. [Using telehomecare to improve chronic disease monitoring]. Sante Publique. 2013 Mar-Apr; 25(2):203-11. French.

43. Kitsiou S, Paré G, Jaana M. Systematic reviews and meta-analyses of home telemonitoring interventions for patients with chronic diseases: a critical assessment of their methodological quality. J Med Internet Res. 2013;15(7):e150.
44. Burkow TM, Vognild LK, Østengen G, Johnsen E, Risberg MJ, Bratvold A, Hagen T, Brattvoll M, Krogstad T, Hjalmarsen A. Internet-enabled pulmonary rehabilitation and diabetes education in group settings at home: a preliminary study of patient acceptability. BMC Med Inform Decis Mak. 2013;5(13):33.

45. Holland A. Telehealth reduces hospital admission rates in patients with COPD. J Physiother. 2013;59(2):129.

46. - Thijssing L, van der Heijden JP, Chavannes NH, Melissant CF, Jaspers MW, Witkamp L. Telepulmonology: effect on quality and efficiency of care. Respir Med. 2014;108(2):314-8. In this population, telemedicine was able to provide support to primary care and avoid unnecessary specialist referrals for COPD patients. However, at the same time, a subset of patients received referrals that otherwise would not have occurred in the absense of telemedicine, arguing that telemedicine may help identify patients who merit specialist referrals with greater precision.

47. Bedra M, McNabney M, Stiassny D, Nicholas J, Finkelstein J. Defining patient-centered characteristics of a telerehabilitation system for patients with COPD. Stud Health Technol Inform. 2013;190:24-6.

48. Jakobsen A, Laursen LC, Ostergaard B, Rydahl-Hansen S, Phanareth KV. Hospital-admitted COPD patients treated at home using telemedicine technology in The Virtual Hospital Trial: methods of a randomized effectiveness trial. Trials. 2013;14(1):280.

49. Almagro P, Castro A. Helping COPD patients change health behavior in order to improve their quality of life. Int $\mathrm{J}$ Chronic Obstr Pulm Dis. 2013;8:335-45.

50. Cheung A, Janssen A, Amft O, Wouters EF, Spruit MA. Usability of digital media in patients with COPD: a pilot study. Int J Technol Assess Health Care. 2013;29(2):162-5.

51. Hæsum LK, Ehlers L, Hejlesen OK. Telehomecare technologies enhance self-management and empowerment among patients with chronic obstructive pulmonary disease (COPD) - where does health literacy fit into this equation? Stud Health Technol Inform. 2013;192:1182.

52. Dinesen B, Huniche L, Toft E. Attitudes of COPD patients towards tele-rehabilitation: a cross-sector case study. Int J Environ Res Public Health. 2013;10(11):6184-98.

53. Voncken-Brewster V, Moser A, van der Weijden T, Nagykaldi Z, de Vries H, Tange H. Usability evaluation of an online, tailored self-management intervention for chronic obstructive pulmonary disease patients incorporating behavior change techniques. JMIR Res Protoc. 2013;2(1):e3.

54. Bjorner JB, Rose M, Gandek B, Stone AA, Junghaenel DU, Ware JE Jr. Difference in method of administration did not significantly impact item response: an IRT-based analysis from the patientreported outcomes measurement information system (PROMIS) initiative. Qual Life Res. 2014;23(1):217-27.

55. Nguyen HQ, Donesky D, Reinke LF, Wolpin S, Chyall L, Benditt JO, Paul SM, Carrieri-Kohlman V. Internet-based dyspnea selfmanagement support for patients with chronic obstructive pulmonary disease. J Pain Symptom Manag. 2013;46(1):43-55.

56. $\bullet$ Global strategy for the diagnosis, management and prevention of COPD, Global Initiative for Chronic Obstructive Lung Disease (GOLD) 2014. Available from: http://www.goldcopd.org/ uploads/users/files/GOLD_Report2014_Feb07.pdf. Accessed on 7 April 2014. This is a large consensus document for the diagnosis and management of COPD, including emerging topics such as telemedicine. It discourages adoption of telemedicine. 